\title{
Retention of flexible intramedullary nails following treatment of pediatric femur fractures
}

\author{
Saam Morshed • Marcie Humphrey • Luis A. Corrales • \\ Meredith Millett $\cdot$ Scott A. Hoffinger
}

Received: 25 August 2006 / Published online: 20 January 2007

(C) Springer-Verlag 2007

\begin{abstract}
Introduction Flexible intramedullary nails are commonly used for the treatment of diaphyseal femur fractures in children. Although, their removal after fracture healing is advocated by some, there are no definitive studies to support the routine removal of these implants. The purpose of this study is to determine the natural history of children with diaphyseal fractures of the femur treated with flexible intramedullary nails and no scheduled nail removal.

Material and methods We performed a retrospective case series of 24 consecutive children treated at our tertiary pediatric referral center for closed diaphyseal femur fractures. All children had intramedullary fixation with flexible titanium nails. The main outcomes measured are fracture healing, incidence of hardware removal, and pain assessment with the use of a followup telephone questionnaire.

Results All the patients healed their fractures. The average follow-up time was 3.6 years. A total of six patients had removal of nails for any reason at an average of 15 months post-injury. The survivorship free of revision due to persistent pain was $72 \%$ at 5 years of
\end{abstract}

S. Morshed $(\varangle) \cdot$ L. A. Corrales

Department of Orthopaedic Surgery,

University of California, San Francisco School of Medicine,

500 Parnassus Avenue, MU-320W, San Francisco,

CA 94143, USA

e-mail: morsheds@orthosurg.ucsf.edu

M. Humphrey · M. Millett · S. A. Hoffinger

Department of Orthopaedic Surgery,

Children's Hospital and Research Center at Oakland,

Oakland, USA follow-up. Twenty-two patients were reached by phone for a final follow-up questionnaire. There was no difference in reports of residual symptoms of pain among those who did have nails removed and those who did $\operatorname{not}(P=0.626)$.

Conclusions Among children with femur fractures treated with flexible intramedullary nailing without scheduled implant removal, about a quarter may ultimately require a second procedure for nail removal due to persistent discomfort. Moreover, up to half of patients can have residual non-debilitating pain at 25 years post-injury regardless of presence or absence of the implant. Whether this is a previously unrecognized adverse outcome of this injury or treatment approach, or due to routinely leaving nails in will have to be assessed in future controlled trials.

Keywords Pediatric $\cdot$ Femur fracture $\cdot$ Titanium . Bone nail $\cdot$ Treatment outcome

\section{Introduction}

Flexible intramedullary nailing is a common procedure used to treat diaphyseal femur fractures in children between the ages of 5 and 16 with excellent outcomes reported $[4-6,8,13,15]$. The main complication of this procedure is pain and soft tissue irritation by the extraosseous portion of the nail tip at the nail insertion site $[7,13,14]$. Several studies have been published detailing the surgical technique, which has typically called for nail removal upon fracture healing at 6 months to 1 year following surgery $[5,6]$.

In the absence of natural history studies to support the routine removal of these implants after healing in 
children, we sought to review the experience at our institution where flexible nails have been cut short and not routinely removed in order to investigate the safety of this approach. A definitive answer to this question could only come from decades of follow-up, which is not currently available given the relatively recent mainstream adoption of this technique. Nevertheless, this study was undertaken with the belief that early follow-up data focused on symptoms of pain and functional limitation and requirements for further surgery at $1-5$ years post-injury is of significance in providing evidence on nail retention as a treatment option for children with these injuries. The purpose of this study is to describe the natural history of treatment of pediatric femur fractures with retention of flexible intramedullary nails as measured by need for further surgery and residual symptoms.

\section{Materials and methods}

A retrospective study was performed of children between the ages of 5 and 13 with closed diaphyseal femur fractures treated at our tertiary pediatric referral center between October 1999 and March 2004, under approval from our institution's committee on human research. During this period, 48 children were treated of which 37 (38 fractures) underwent internal fixation with flexible intramedullary nails. Of the 11 excluded, 6 were less than 7 years of age and underwent spica casting and 5 were teenagers treated with rigid intramedullary nails. Children with an underlying neuromuscular disease (cerebral palsy or myelomeningocele), a metabolic bone disorder, or pathologic fracture were also excluded, as were those with less than 1 year of followup. This left a consecutive series of 24 children ( 25 fractures) for inclusion in this study.

Surgeries were performed by one of four full-time fellowship trained pediatric orthopaedic faculty at our institution. Synthes (West Chester, PA, USA) flexible titanium nails were used in all cases. Standard technique for implantation of titanium elastic nails was used as described elsewhere [5, 6]. Implant size was chosen by measuring $40 \%$ of the narrowest diameter of the femoral diaphysis. Two to three titanium elastic nails were placed in a retrograde fashion through the distal part of the femur depending on fracture pattern and stability. An attempt was made to cut all nails so they would lie flush against the femoral cortex, with no more than $1-1.5 \mathrm{~cm}$ protruding from the bone, with the intention that they would not be removed in all cases. Pre-operatively and post-operatively all patients were treated with IV and PO narcotics. No patient was dis- charged without a filled prescription for oral pain medications.

Post-operative management consisted of physical therapy with touchdown weight bearing as soon as the child was comfortable. Gentle knee exercises and quadriceps strengthening were begun shortly thereafter with advancement to full weight bearing usually by 6 weeks. At each post-injury visit patients underwent radiographic examination with assessment of alignment, callus formation, and the status of the implants. Clinical examination included assessment of limb lengths, strength, range of motion, alignment and rotation, condition of the wound and skin, and pain. Patients were followed clinically until the fracture had healed and they had returned to full activity. Any complication was recorded. Children with persistent symptoms of irritation at the nail insertion site beyond the period of fracture healing were offered nail removal only after 6 months post-injury and the ability to bare weight.

At the time of chart review for this study, an attempt was made to locate all patients included in the study for interview including those still following up in our clinic. Twenty-two patients with 23 femoral fractures (92\% follow-up) were successfully contacted by phone for a detailed follow-up questionnaire by a research nurse who was not involved in clinical care of the patient and blinded to the details of the child's medical history. Patients and a caregiver were asked questions regarding the presence or absence of pain (scale from 1 to 10), incidence of further complications or requirement of a second surgery to remove the nails, and any changes in pain or functional status following a second procedure (See Appendix).

Data analysis was undertaken using STATA version 8.2 (College Station, TX). All statistics considered femora as the unit of analysis rather than patients. The non-parametric Wilcoxon rank-sum test was used to test differences in mean pain score and the Fisher's exact test was used to assess the association between pain and whether or not the nails were still in place at the time of the follow-up questionnaire as well as to assess multiple potential risk factors for nail removal including gender, age at time of injury, number of rods used or fracture pattern. Significance was set at a $P$ value less than 0.05 . Any significantly associated predictors of nail removal in bivariate analysis were entered in to a multivariate logistic regression model. Kaplan-Meier survivorship analysis was used to calculate survivorship. Failure events were counted as requirement for nail removal for any reason and for removal of nails due to persistent pain or irritation. 


\section{Results}

Average age at fracture for the 24 children included in this review was 9.2 years (range 6.5-13 years). There were four girls and twenty boys in this series. Average follow-up was 3.6 years (range 1.4-4.8 years). All fractures were closed. All children healed their fractures without need for any additional procedures. There were no intra-operative complications, infections, wound healing problems, or neurological injuries. No patient ended up with angulation or length inequality that exceeded acceptable published guidelines [12] and there were no refractures. No patient was using medications for pain by the time the fracture had healed or at their most recent follow-up. Individual patient data is summarized in Table 1.

At the time of most recent follow-up or questionnaire, six of the twenty-four patients ( 7 of 25 fractures) had undergone rod removal without complications. Kaplan-Meier survivorship without nail removal for any reason was $68 \%$ (95\% CI 44-84\%) at mean followup of 35 months and at 5 years (Fig. 1). Figure $2 a, b$ shows a patient without hardware removal at 2.4 years of follow-up. One of the rod removals was performed at an outside facility at 1 year post-injury in a child who

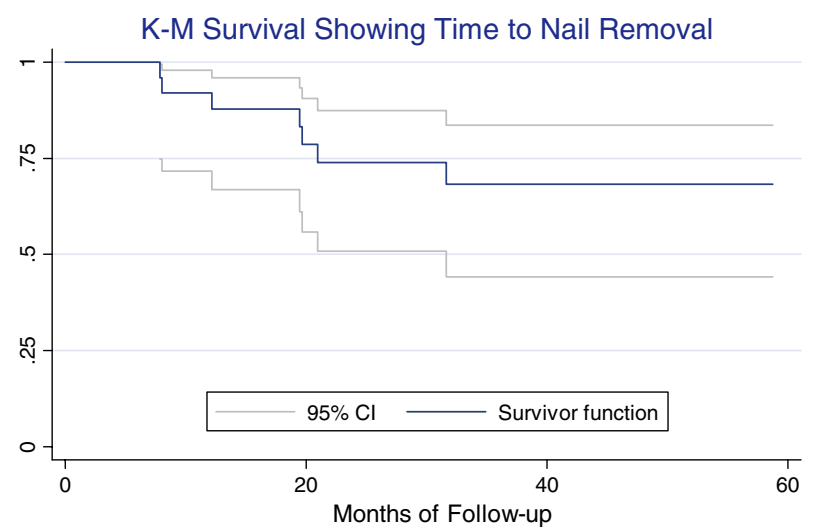

Fig. 1 Kaplan-Meier survivorship analysis illustrating time to failure defined as second surgery for nail removal for any reason

was asymptomatic, all other were removed secondary to persistent local symptoms of pain and irritation. Survivorship without nail removal for complaints of insertion site irritation was $72 \%$ (95\% CI $47-87 \%)$. Average time of nail removal in those six patients was 1.26 year (0.6-2.75 years).

We were also interested in the presence or absence of discomfort, and whether we could determine if this discomfort was related to the removal of the implants.

Table 1 Individual patient data

\begin{tabular}{|c|c|c|c|c|c|c|c|c|c|c|c|c|c|c|}
\hline Patient & Gender & $\begin{array}{l}\text { Age } \\
\text { (years) }\end{array}$ & Side & $\begin{array}{l}\text { Fracture } \\
\text { pattern }\end{array}$ & Location & $\begin{array}{l}\text { Number } \\
\text { of nails }\end{array}$ & $\begin{array}{l}\text { Size } \\
\text { of nails } \\
(\mathrm{mm})\end{array}$ & Questionnaire & $\begin{array}{l}\text { Nails } \\
\text { removed }\end{array}$ & $\begin{array}{l}\text { Reason } \\
\text { for } \\
\text { removal }\end{array}$ & $\begin{array}{l}\text { Time to } \\
\text { removal } \\
\text { (years) }\end{array}$ & $\begin{array}{l}\text { Current } \\
\text { pain }\end{array}$ & $\begin{array}{l}\text { Pain } \\
\text { score }\end{array}$ & $\begin{array}{l}\text { Follow-up } \\
\text { (years) }\end{array}$ \\
\hline 1 & M & 7.8 & $\mathrm{~L}$ & Oblique & MT & 2 & 3 & No & No & NA & NA & No & 0 & 1.4 \\
\hline 2 & M & 10.0 & $\mathrm{~L}$ & Oblique & MT & 2 & 4.5 & Yes & No & NA & NA & No & 0 & 4.6 \\
\hline 3 & $\mathrm{~F}$ & 9.2 & $\mathrm{R}$ & Transverse & MT & 2 & 3.5 & Yes & No & NA & NA & No & 0 & 4.8 \\
\hline 4 & M & 9.5 & $\mathrm{R}$ & Segmental & PT & 2 & 4 & Yes & No & NA & NA & No & 0 & 3.6 \\
\hline 5 & M & 12.0 & $\mathrm{R}$ & Transverse & MT & 2 & 3.5 & Yes & No & NA & NA & No & 0 & 4.9 \\
\hline 6 & $\mathrm{~F}$ & 7.9 & $\mathrm{R}$ & Transverse & MT & 2 & 3 & Yes & No & NA & NA & No & 0 & 4.3 \\
\hline 7 & M & 8.5 & $\mathrm{R}$ & Transverse & MT & 2 & 3 & Yes & No & NA & NA & No & 0 & 4.2 \\
\hline 8 & M & 10.3 & $\mathrm{R}$ & Transverse & PT & 2 & 3 & Yes & No & NA & NA & No & 0 & 3.8 \\
\hline 9 & M & 7.4 & $\mathrm{~L}$ & Oblique & DT & 2 & 3 & Yes & No & NA & NA & No & 0 & 3.4 \\
\hline 10 & M & 10.3 & $\mathrm{R}$ & Transverse & MT & 2 & 4 & Yes & Yes & Pain & 1.6 & No & 0 & 3.6 \\
\hline 10 & M & 10.3 & $\mathrm{~L}$ & Transverse & DT & 2 & 4 & Yes & Yes & Pain & 1.6 & No & 0 & 3.6 \\
\hline 11 & M & 6.6 & $\mathrm{R}$ & Oblique & MT & 2 & 3 & Yes & Yes & Pain & 0.6 & Yes & 2 & 3.7 \\
\hline 12 & M & 9.5 & $\mathrm{~L}$ & Spiral & MT & 2 & 3.5 & Yes & No & NA & NA & No & 0 & 4.3 \\
\hline 13 & $\mathrm{~F}$ & 9.1 & $\mathrm{~L}$ & Oblique & MT & 2 & 3.5 & Yes & Yes & Pain & 0.7 & Yes & 8.5 & 2.4 \\
\hline 14 & M & 9.1 & $\mathrm{~L}$ & Spiral & MT & 2 & 3.5 & Yes & Yes & No pain & 0.6 & No & 0 & 2.8 \\
\hline 15 & M & 7.6 & $\mathrm{R}$ & Segmental & PT & 3 & 4 & Yes & No & NA & NA & Yes & 1 & 1.4 \\
\hline 16 & M & 13.0 & $\mathrm{R}$ & Spiral & PT & 2 & 3.5 & Yes & Yes & Pain & 2.8 & No & 0 & 3.3 \\
\hline 17 & M & 7.6 & $\mathrm{R}$ & Spiral & PT & 2 & 3 & No & No & NA & NA & No & 0 & 1.86 \\
\hline 18 & M & 11.1 & $\mathrm{~L}$ & Transverse & PT & 2 & 4 & Yes & No & NA & NA & No & 0 & 4.36 \\
\hline 19 & $\mathrm{~F}$ & 6.5 & $\mathrm{R}$ & Transverse & DT & 2 & 3 & Yes & No & NA & NA & Yes & 5 & 4.2 \\
\hline 20 & M & 8.1 & $\mathrm{R}$ & Spiral & MT & 2 & 3 & Yes & No & NA & NA & Yes & 2 & 3 \\
\hline 21 & M & 11.1 & $\mathrm{~L}$ & Oblique & PT & 2 & 4 & Yes & No & NA & NA & No & 0 & 4 \\
\hline 22 & M & 10.4 & $\mathrm{R}$ & Oblique & MT & 2 & 3.5 & Yes & Yes & Pain & 1 & Yes & 5 & 3.2 \\
\hline 23 & M & 10.2 & $\mathrm{~L}$ & Transverse & MT & 2 & 3.5 & Yes & No & NA & NA & Yes & 5 & 3.7 \\
\hline 24 & M & 6.7 & $\mathrm{~L}$ & Transverse & MT & 2 & 3 & Yes & No & NA & NA & No & 0 & 5.04 \\
\hline
\end{tabular}

Telephone questionnaire completed: yes or no, time to most recent clinic visit or telephone interview

$M T$ middle third, $P T$ proximal third, $D T$ distal third 
Fig. 2 a, b AP and lateral $\mathrm{X}$-ray of right femur from 12 year old boy 2.4 years after implantation of flexible nails for a mid-shaft fracture
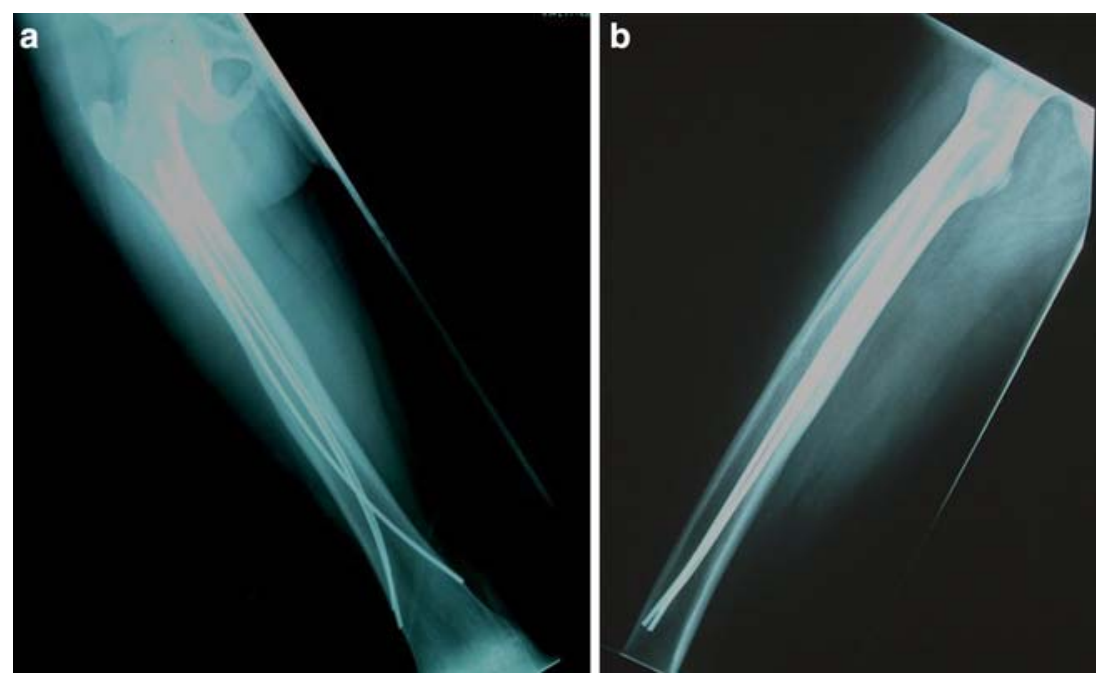

Seven of the twenty-two subjects successfully contacted reported persistent painful symptoms with an average pain score of 4.1 with no difference between those who had their implants removed and those who did not $(P=0.35)$. Most patients described their symptoms as "achy" occasionally causing a slight limp, but not interfering with routine activities. Of the 22 patients that were contacted for detailed interview, six had had their nails removed (seven femora). Of these six patients, three reported continued thigh discomfort even after nail removal (43\% of femora). Of the 16 patients with rods in place, four reported thigh discomfort (25\% of femora). This difference was not significant $(P=0.626)$. All patients who had their rods removed due to pain reported improvement in local irritation following nail removal.

We were not able to accurately correlate the need for nail removal and prominence of the tip of the nail as assessed on routine radiographs due to the variable angle at which the films were taken and projection artifact. Also, no significant associations were found between nail removal and gender, age at time of injury, number of nails used or fracture pattern.

\section{Discussion}

We have reported a consecutive series of 24 school age children (25 femora) who underwent internal fixation of a diaphyseal femur fracture with flexible titanium nails in whom no implant removal was planned. All patients healed their fractures without incidence of significant malunion, length discrepancy, or refracture. Seventy-two percent of patients did not require removal of the nails due to pain, local irritation or other complaints at an average follow-up of almost
3 years. Most children who did require hardware removal underwent a second surgery between 1 and 2 years post-injury.

A surprising finding was the high prevalence of knee and thigh pain as assessed by a follow-up telephone interview at up to 5 years post-injury. Seven of twentytwo children contacted for recent follow-up complained of pain, regardless of whether the nails had been removed or not. This occurred despite returning to full activity and in the absence of other complaints possibly suggesting an overly sensitive questionnaire for these symptoms. It may also be that this high proportion of patients with residual pain is due to retained hardware in this cohort of patients. Whether or not hardware retention or delay removal causes these symptoms and how clinically significant they actually are can only be validly assessed with a controlled study.

Routine hardware removal in the pediatric population has been questioned by Kahle and others due to complication rates as high as $14 \%[10,11]$. In a recent series by Luhmann [14] on complications associated with the use of titanium elastic nails for pediatric femoral shaft fractures, one of two major complications was knee joint sepsis following hardware removal. In addition, other reasons for objection to hardware removal in children are the second surgery with the attendant discomfort, inconvenience, time off school and work, and temporary restriction from play.

Another downside to routine hardware removal is the additional resources consumed. There is little data in the literature looking at cost-effectiveness of hardware retention or removal following fracture fixation of long bones in children. In a study from Finland, data on all orthopaedic operations performed during a 7 year period were collected at one institution and removal of implants was found to account for nearly 
one third of all elective surgeries, and $15 \%$ of all operations [3]. The removal policy and practice of internal fixation devices vary between institutions but it can be inferred that such surgeries consume hospital resources and may potentially not be cost-effective. In a study looking at absorbable fracture fixation devices that obviate future implant removal, substantial cost-savings could be achieved depending on baseline rates of hardware removal [2]. We did not assess cost in this study and future prospective studies are needed to accurately assess cost-effectiveness.

The rationale for routine hardware removal includes issues such as local irritation, stress shielding, growth issues (acceleration, arrest), and difficulty should later removal become necessary $[1,16]$. Because this is a weight-sharing device, there should be minimal stress shielding. Moreover, refracture has only been reported in cases where nails were prematurely removed [5, 7]. Physeal damage could be caused by the initial trauma or potentially nail insertion, but provided the nails are in the recommended location, there should be no growth issues incurred by leaving the nails in place. Finally, there is data suggesting that titanium implants used in internal fixation of long bone fractures can lead to an immuno-inflammatory reaction [17]. The clinical importance of such a reaction is unknown, though patients should be given the best available data regarding the possible bioactivity of the implants used. While the question of removal of flexible nails after pediatric femur fractures remains controversial, no data exists to show adverse consequences of their being left in place.

We find the main concern with hardware retention to be later difficulty removing the nails should the patient suffer a second femur fracture. This would indeed present some challenge, as the flexible nails would need to be removed to allow placement of a rigid femoral nail. While femur fractures are one of the most common long bone fractures in children, they are rare overall with reported incidence in the United States of 19.15 per 100,000 , varying significantly with age, gender and race [9]. This puts the risk to any one individual at 1:5,000, and if considered independently, the risk of a second femur fracture would be far lower. Specific study of the risk of a second femur fracture has not been reported to our knowledge in children or adults and is difficult to estimate accurately. Decision analysis or cost-effectiveness analysis may be a useful tool in informing decision making in the future.

This study has several limitations including lack of a control group, incomplete follow-up on telephone surveys, and small sample size making it difficult to assess for potential risk factors for nail removal and other chronic symptoms. This is a descriptive study of the experience at one pediatric referral center undergoing a single treatment strategy. Inference as to the superiority of this versus other strategies cannot be made without a control group. In the future, a multicenter prospective controlled observational study or randomized trial will be needed to more validly answer questions raised here about routine nail removal following operative fixation of pediatric femur fractures. Still, this case series is the first to our knowledge to report the early results of nail retention as an alternative to uniform removal and provides important information for future studies that compare these two treatment options.

\section{Conclusions}

We have described the early natural history of retained flexible intramedullary nails following the treatment of pediatric femoral shaft fractures. We have found that by cutting the nails flush to lie against the metaphyseal flare of the femoral condyle, nail removal is unnecessary in over $70 \%$ of our patients. We suggest this strategy as an alternative to routine rod removal following flexible intramedullary nailing for femur fractures in the pediatric population in order to avoid the inconvenience to patients and potential complications of a second surgical procedure. Further research will be necessary to corroborate or refute this practice and the relative risk of symptoms of pain compared to patients who undergo routine nail removal.

Acknowledgments Dr. Morshed has received educational and research funding from the Orthopaedic Research and Education Foundation Clinical Research Training Fellowship. Luis A. Corrales received educational funding from the Doris Duke Clinical Research Fellowship Program. This study was supported in part by the Children's Hospital Oakland Pediatric Clinical Research Center M01 RR01271.

\section{Appendix: Telephone-based questionnaire}

1. Does your child have pain now in the operated leg? Yes or No

2. If your child currently has pain, please rate it on a scale from 0 (no pain) to 10 (worst pain imaginable).

3. If your child currently has pain, is his or her pain located in the knee, surgical site, thigh, or somewhere else?

4. Does your child's operated leg appear different in strength compared with the other leg?

Yes or No 
5. Did your child require any further surgery on that leg after the implants were placed?

Yes or No

A. If yes, when?

B. If yes, what?

C. If yes, why?

6. If the nails were removed, did your child's symptoms improve following this additional procedure? Yes or No

7. Are there any other problems or limitations associated with the broken leg?

\section{References}

1. Alzahrani AG, Behairy YM, Alhossan MH, Arab FS, Alammari AA (2003) Removal of internal fixation in pediatric patients. Saudi Med J 24(3):254-255

2. Bostman OM (1996) Metallic or absorbable fracture fixation devices. A cost minimization analysis. Clin Orthop Relat Res 329:233-239

3. Bostman O, Pihlajamaki H (1996) Routine implant removal after fracture surgery: a potentially reducible consumer of hospital resources in trauma units. J Trauma 41(5):846-849

4. Cramer KE, Tornetta P 3rd, Spero CR, Alter S, Miraliakbar H, Teefey J (2000) Ender rod fixation of femoral shaft fractures in children. Clin Orthop Relat Res 376:119-123

5. Flynn JM, Hresko T, Reynolds RA, Blasier RD, Davidson R, Kasser J (2001) Titanium elastic nails for pediatric femur fractures: a multicenter study of early results with analysis of complications. J Pediatr Orthop 21(1):4-8

6. Flynn JM, Luedtke L, Ganley TJ, Pill SG (2002) Titanium elastic nails for pediatric femur fractures: lessons from the learning curve. Am J Orthop 31(2):71-74
7. Flynn JM et al (2004) Comparison of titanium elastic nails with traction and a spica cast to treat femoral fractures in children. J Bone Joint Surg Am 86-A(4):770-777

8. Heinrich SD, Drvaric DM, Darr K, MacEwen GD (1994) The operative stabilization of pediatric diaphyseal femur fractures with flexible intramedullary nails: a prospective analysis. J Pediatr Orthop 14(4):501-507

9. Hinton RY, Lincoln A, Crockett MM, Sponseller P, Smith G (1999) Fractures of the femoral shaft in children. Incidence, mechanisms, and sociodemographic risk factors. J Bone Joint Surg Am 81(4):500-509

10. Jago ER, Hindley CJ (1998) The removal of metalwork in children. Injury 29(6):439-441

11. Kahle WK (1994) The case against routine metal removal. J Pediatr Orthop 14(2):229-237

12. Kasser JR, Beaty JH (2001) Femoral shaft fractures. In: Beaty JH, Kasser JR (eds) Rockwood, and Wilkins' fractures in children. Lippincott/Williams \& Wilkins, Philadelphia, p 948

13. Ligier JN, Metaizeau JP, Prevot J, Lascombes P (1988) Elastic stable intramedullary nailing of femoral shaft fractures in children. J Bone Joint Surg Br 70(1):74-77

14. Luhmann SJ, Schootman M, Schoenecker PL, Dobbs MB, Gordon JE (2003) Complications of titanium elastic nails for pediatric femoral shaft fractures. J Pediatr Orthop 23(4):443447

15. Sanders JO, Browne RH, Mooney JF, Raney EM, Horn BD, Anderson DJ Hennrikus WL, Robertson WW (2001) Treatment of femoral fractures in children by pediatric orthopedists: results of a 1998 survey. J Pediatr Orthop 21(4):436-441

16. Schmalzried TP, Grogan TJ, Neumeier PA, Dorey FJ (1991) Metal removal in a pediatric population: benign procedure or necessary evil? J Pediatr Orthop 11(1):72-76

17. Voggenreiter G, Leiting S, Brauer H, Leiting P, Majetschack M, Bardenheuer M, Obertacke U (2003) Immuno-inflammatory tissue reaction to stainless-steel and titanium plates used for internal fixation of long bones. Biomaterials 24:247-254 